\title{
Application of poloidal beta and plasma internal inductance in determination of input power time of Damavand tokamak
}

\author{
Ehsanallah Noori $^{1} \cdot$ Yahya Sadeghi $^{1} \cdot$ Mahmood Ghoranneviss $^{2}$
}

Received: 20 April 2016/ Accepted: 27 September 2016/Published online: 7 October 2016

(c) The Author(s) 2016. This article is published with open access at Springerlink.com

\begin{abstract}
In this study, magnetic measurement of poloidal fields were used to determine poloidal beta and plasma internal inductance of Damavand tokamak combination of poloidal beta and plasma internal inductance $\left(\beta_{p}+\frac{l_{i}}{2}\right)$, known as Shafranov parameter, was obtained experimentally in terms of normal and tangential components of the magnetic field. Plasma internal inductance and poloidal beta were obtained using parametrization method based on analytical solution of Grad-Shafranov equation (GSE) and compared with parabolic-like profile of toroidal current density approach for determination of the plasma internal inductance. Finding evolution of $\beta_{p}+\frac{l_{i}}{2}$ and plasma internal inductance. Finding poloidal beta (Shafranov parameter and internal inductance) and using energy balance equation, thermal energy and energy confinement were determined qualitatively in terms of poloidal beta during a regular discharge of Damavand tokamak.
\end{abstract}

Keywords Damavand tokamak - Magnetic measurements Plasma inductance . Poloidal beta

Ehsanallah Noori

ehssan.noori@gmail.com

1 Plasma Physics and Nuclear Fusion Research School, Nuclear Science and Technology Research Institute (NSTRI), Tehran, Iran

2 Plasma Physics Research Center (PPRC), Science and Research Branch, Islamic Azad University, Tehran, Iran

\section{Introduction}

Equilibrium parameters of tokamak such as plasma internal inductance $\left(l_{i}\right)$, poloidal beta $\left(\beta_{p}\right)$ or combination expression $\beta_{p}+\frac{l_{i}}{2}$, known as Shafranov parameter, play very crucial roles in characterization of tokamak activities. It is also possible to extract many other plasma parameter such as displacement of plasma column, prediction of vertical displacement event (VDE) and energy confinement time of tokamak using equilibrium parameters [4-7]. In tokamaks with circular cross section, it is possible to find evolution of the Shafranov parameter using model which is based on expansion (with respect to the inverse aspect ratio parameter of the tokamak) of polidal flux function $\psi$ and the first order solution of the well known Grad-Shafranov equation (GSE) in toroidal coordinate system [8-10]. This approach (the first order solution of the poloidal flux function and finding the first order poloidal and radial components of the magnetic field), however, is an approximate method which provide reliable information about Shafranov parameter and horizontal displacement of the plasma column (Shafranov shift) in tokamaks with circular cross section or when the non-circularity of the plasma surface is considered as a correction factor. Therefore, this model becomes inaccurate when the plasma non-circularity increases and does not provide valid assessment about Shafranov parameter in elongated tokamaks. In non-circular, highly elongated tokamaks, the plasma internal inductance and poloidal beta can be determined separately while for circular ones, only combination of $l_{i}$ and $\beta_{p},\left(\beta_{p}+\frac{l_{i}}{2}\right)$, can be obtained and separation of $l_{i}$ and $\beta_{p}$ can be made using diamagnetic flux measurement, in addition to magnetic diagnostics. This approach, however, makes significant 
fluctuations and noise in small size tokamaks and there are some difficulties in compensation of the flux differences using diamagnetic flux measurement technique in circular and non-circular tokamaks. Therefore, it is highly desirable to use an effective, independent method to determine $\beta_{p}$ and $l_{i}$ separately from the external poloidal magnetic measurements alone (independent of the diamagnetic flux measurement).

In general, determination of Shafranov parameter relies on magnetic measurements that are conducted by magnetic probes which are mounted around the plasma surface. In large aspect ratio tokamaks, it is possible to find poloidal beta and plasma internal inductance from Shafranov parameter using axillary diagnostic facilities such as diamagnetic flux measurements [11-14]. However, some other equilibrium parameters such as plasma edge safety factor and poloidal flux function can be obtained directly from calculation of Shafranov parameter [15-17]. Having evolution of the Shafranov parameter, plasma internal inductance (poloidal beta) can be found by definition of toroidal current density or using parametrization method, based on analytical solution of Grad-Shafranov equation (GSE) which describes magnetohydrodynamics (MHD) equilibrium of axi-symmetric configurations such as tokamak.

To achieve the thermonuclear condition $\left(\bar{n} \tau_{E} \bar{T}>5 \times\right.$ $10^{21} \mathrm{~m}^{-3} \mathrm{~s} \mathrm{keV}$ ) in magnetically confined configurations such as tokamak, it is necessary to maintain plasma for sufficient time. Therefore, input power time (energy confinement time) is one of the most important parameters of the plasma ignition in tokamaks [1-3]. In general, input power time should be analyzed by microscopic and transport behavior of the plasma. The maximum energy confinement time can be determined by the microscopic behavior of the plasma such as collisions and microinstabilities. This behavior ultimately leads to macroscopic energy transport, which can be either classical or anomalous depending on the processes involved. In the absence of instabilities, the confinement of toroidally symmetric tokamak plasma is determined by Coulomb collisions. Since these phenomena require a knowledge of individual particles motion on short length scales and time scales, they are usually treated by kinetic models, but including only limited geometry because of the complexity of the physics.

In this paper, magnetic measurements method is used to find equilibrium parameters (Shafranov parameter, internal inductance and poloidal beta) of Damavand tokamak. Finding evolution of the Shafranov parameter in typical discharge of Damavand tokamak, plasma internal inductance (poloidal beta) is determined using analytic solution of Grad-Shafranov equation and is compared with the semi-analytical determination of the plasma internal inductance using parabilic-kike profile of the toroidal current density. Having poloidal beta, power balance equation is used to find thermal energy of the plasma and global energy confinement time in terms of poloidal beta during a regular discharge of Damavand tokamak.

\section{Physical description of energy confinement time and equilibrium parameters of elongated tokamaks}

To maintain thermonuclear temperatures in tokamaks, there should be an energy balance between heat sources and power losses. The total energy balance of an Ohmically heated tokamak is defined as $[1,2]$

$\frac{d U}{d t}=\frac{P_{\text {Ohmic }}}{V_{p}}-\frac{U}{\tau_{E}}$

where $V_{p}$ and $P_{\text {Ohmic }}$ are volume and input power due to Ohmic heating of the plasma, respectively. The parameter $\tau_{E}$ is introduced as input power (energy confinement) time which determines plasma confinement duration. The total thermal energy density of the plasma can be expressed in term of volume-averaged pressure as [18]

$U=\frac{3}{2}\langle P\rangle_{V}=\frac{3}{2} \beta_{p} \frac{\left\langle B_{p}\right\rangle_{L}^{2}}{2 \mu_{0}}$

where the volume averaged pressure of the plasma is introduced as

$\langle P\rangle_{V} \equiv \frac{\int P d \mathbf{r}}{\int d \mathbf{r}}=\frac{\beta_{p}\left\langle B_{p}\right\rangle_{L}^{2}}{2 \mu_{0}}$

$\left\langle B_{p}\right\rangle_{L}=\frac{\oint B_{p} d l_{p}}{\oint d l_{p}}$ is defined as line averaged poloidal magnetic field on the plasma surface. The input power can be written as [19]

$P_{\text {Ohmic }} \equiv V_{R} I_{p}=V_{L} I_{p}-\frac{d}{d t}\left(\frac{1}{2} L I_{p}^{2}\right)$

where $V_{R}$ and $V_{L}$ are resistive and toroidal loop voltages and $L$ is the total inductance which is defined as

$L=\mu_{0} R_{0}\left[\ln \left(\frac{8 R_{0}}{a}\right)+\frac{l_{i}}{2}-2\right]$

the dimensionless parameter $l_{i}$ is introduced as plasma internal inductance per unit length which is defined as

$l_{i}=\frac{\left\langle B_{p}^{2}\right\rangle}{\left\langle B_{p}\right\rangle_{L}^{2}}$ 
Static equilibrium of axi-symmetric toroidal configurations such as tokamak, is mainly described by well known GradShafranov equation (GSE) which in cylindrical coordinate system can be written as $[1,20,21]$

$$
\begin{aligned}
R \frac{\partial}{\partial R}\left(\frac{1}{R} \frac{\partial \psi}{\partial R}\right)+\frac{\partial^{2} \psi}{\partial Z^{2}} & =-\mu_{0} R^{2} 4 \pi^{2} \frac{\partial P}{\partial \psi}-4 \pi^{2} F \frac{\partial F}{\partial \psi} \\
& =-\mu_{0} 2 \pi R J_{\varphi}(R, Z)
\end{aligned}
$$

where $\psi(R, Z)$ is a scalar function proportional to poloidal magnetic flux

$2 \pi \psi=\int \mathbf{B}_{p} \cdot d \mathbf{s}_{p}$

$P(\psi)$ denotes the plasma pressure and $F(\psi)$ is a free function proportional to toridal current. It would be convenient to write the GSE in dimensionless coordinate as:

$$
\begin{aligned}
x \frac{\partial}{\partial x}\left(\frac{1}{x} \frac{\partial \psi}{\partial x}\right)+\frac{\partial^{2} \psi}{\partial y^{2}} & =-\mu_{0} 4 \pi^{2} R_{0}^{4} x^{2} \frac{d P}{d \psi}-4 \pi^{2} R_{0}^{2} F \frac{d F}{d \psi} \\
& =-\mu_{0} 2 \pi R_{0}^{3} x J_{\varphi}
\end{aligned}
$$

According to Solov'ev model, a simple choice of $P(\psi)$ and $F(\psi)$ are

$$
\begin{aligned}
& \mu_{0} 4 \pi^{2} R_{0}^{4} \frac{d P}{d \psi}=A_{1} \\
& 4 \pi^{2} R_{0}^{4} F \frac{d F}{d \psi}=A_{2}
\end{aligned}
$$

Therefore, the GSE can be rewritten as

$$
x \frac{\partial}{\partial x}\left(\frac{1}{x} \frac{\partial \psi}{\partial x}\right)+\frac{\partial^{2} \psi}{\partial y^{2}}=-\left(A_{1} x^{2}+A_{2}\right)
$$

The solution of equation (10) can be written as

$$
\psi(x, y)=\psi_{h}(x, y)-\frac{A_{1}}{8} x^{4}-\frac{A_{2}}{2} y^{2}
$$

where $\psi_{h}(x, y)$ is the solution of the homogenios equation. For tokamaks with up-down symmetry, homogenios part of the GSE can have polynomial-like solution in which atleast four terms are required for the description of tokamak plasma equilibrium. Therefore, the solution of Eq. 10 is expressed as:

$$
\begin{aligned}
\psi(x, y)= & C_{1}+C_{2} x^{2}+C_{3}\left(x^{4}-4 x^{2} y^{2}\right)+C_{4}\left(x^{2} \ln x-y^{2}\right) \\
& -\frac{A_{1}}{8} x^{4}-\frac{A_{2}}{2} y^{2}
\end{aligned}
$$

Equation 12 is a desired solution of GSE that describes the equilibrium of tokamak plasma with arbitrary cross-section, elongation, triangularity and $\beta$. Coefficient $C_{n}$ are determined from the boundary condition of the plasma surface while particular coefficients $A_{1}$ and $A_{2}$ should be found from experimental parameter of plasma such as plasma current $I_{p}$ and $\beta_{p}+\frac{l_{i}}{2}$ (linear combination of plasma internal inductance and poloidal beta) which is known as Shafranov parameter (asymmetry factor). In other words, the homogeneous coefficients are obtained analytically while particular coefficients $A_{1}$ and $A_{2}$ parameterize the GSE and for this reason, this approach of solving the GSE is recognized as parametrization method. It is considered that the surface of plasma boundary is smooth which is reasonable in Ohmically heated, limiter based tokamaks such as Damavand. To determine unknown coefficients $C_{n}$, magnetic flux function $\psi(x, y)$ and its derivative should be matched at three test points of plasma boundary. These points include: inner equatorial points, outer equatorial points and the highest point of the plasma surface.

The boundary of the plasma surface for an elongated, D-shaped cross-section is given by the parametric equations

$$
\begin{aligned}
& x=1+\varepsilon \cos (\theta+\alpha \sin \theta) \\
& y=\varepsilon \kappa \sin \theta
\end{aligned}
$$

where $\theta$ is the poloidal angle in toroidal coordinate system which varies from 0 to $2 \pi, \kappa$ is introduced as elongation of the cross-section, $\alpha=\sin ^{-1}(\delta)$ defines the triangularity of the plasma surface and $\varepsilon=\frac{a}{R_{0}}$ with $a$ as the horizontal halfwidth of plasma known as plasma radius, is the inverse aspect ratio parameter. Figure 1 shows boundary of the plasma surface and definitions of the geometrical parameters $\varepsilon, \kappa$ and $\delta$. In the case of fixed boundary solution of GSE, the flux function should be constant on the plasma surface. For this reason, $\Psi=0$ is considered on the plasma surface. Therefore, the boundary conditions on the plasma surface are summarized as:

$$
\begin{aligned}
\psi(1-\varepsilon, 0)= & C_{1}+C_{2}(1-\varepsilon)^{2}+C_{3}(1-\varepsilon)^{4}+C_{4}(1-\varepsilon)^{2} \\
& \times \ln (1-\varepsilon)-\frac{A_{1}}{8}(1-\varepsilon)^{4}=0 \\
\psi(1+\varepsilon, 0)= & C_{1}+C_{2}(1+\varepsilon)^{2}+C_{3}(1+\varepsilon)^{4}+C_{4}(1+\varepsilon)^{2} \\
& \times \ln (1+\varepsilon)-\frac{A_{1}}{8}(1+\varepsilon)^{4}=0 \\
\psi(1-\delta \varepsilon, \kappa \varepsilon)= & C_{1}+C_{2}(1-\delta \varepsilon)^{2}+C_{3}\left[(1-\delta \varepsilon)^{4}-4(1-\delta \varepsilon)^{2}(\kappa \varepsilon)^{2}\right] \\
& +C_{4}\left[(1-\delta \varepsilon)^{2} \ln (1-\delta \varepsilon)-(\kappa \varepsilon)^{2}\right]-\frac{A_{1}}{8}(1+\varepsilon)^{4} \\
& -\frac{A_{2}}{2}(\kappa \varepsilon)^{2}=0 \\
\frac{\partial \psi(x, y)}{\partial x} \mid x= & 1-\delta \varepsilon=2 C_{2}+4 C_{3}\left[(1-\delta \varepsilon)^{2}-2(\kappa \varepsilon)^{2}\right] \\
y= & \kappa \varepsilon \\
& +C_{4}(2 \ln (1-\delta \varepsilon)+1)-\frac{A_{1}}{2}(1-\delta \varepsilon)^{2}=0
\end{aligned}
$$




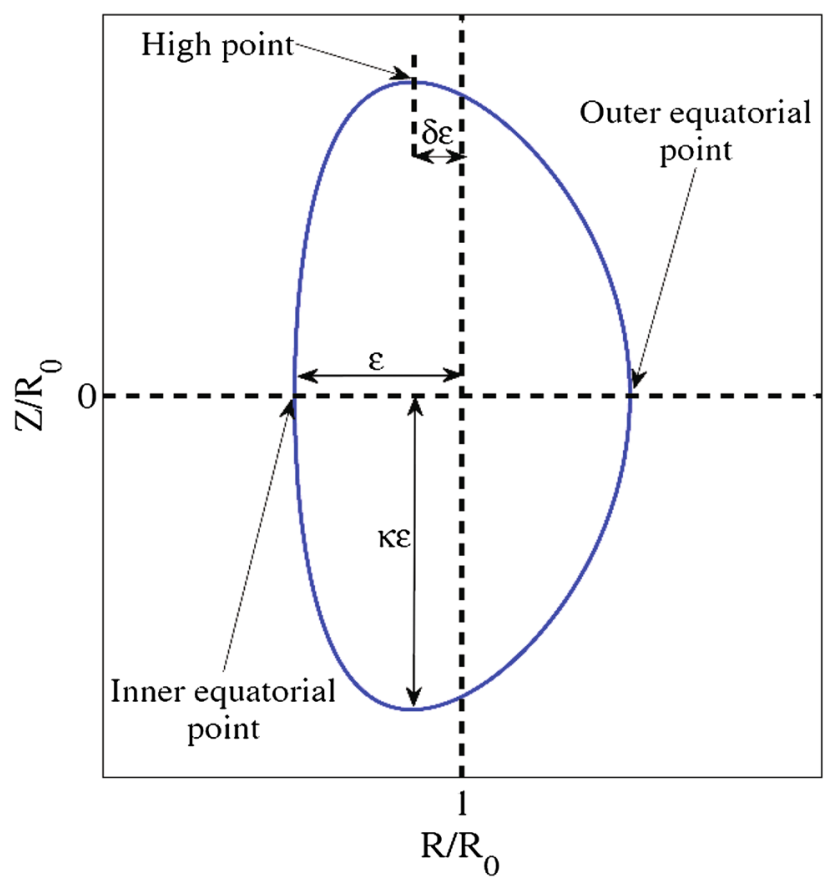

Fig. 1 Geometry view of elongated plasma surface

the last condition of Eq. (17) defines the maximum of the flux function at the highest point of the plasma surface. The two unknown coefficients $A_{1}$ and $A_{2}$ are determined experimentally from the measurement of plasma current and Shafranov parameter as:

$$
\begin{aligned}
& I_{p}=\int J_{\varphi} d R d Z=\frac{1}{2 \pi \mu_{0} R_{0}} \int\left(A_{1} x+\frac{A_{2}}{x}\right) d x d y \\
& \Lambda+1= \beta_{p}+\frac{l_{i}}{2}=\frac{C_{p}^{2}}{\left[\int\left(A_{1} x+\frac{A_{2}}{x}\right) d x d y\right]^{2} V_{p}} \\
& \quad \times\left(2.5 A_{1} \int \Psi(x, y) x d x d y+0.5 A_{2} \int \frac{\Psi(x, y)}{x} d x d y\right)
\end{aligned}
$$

where $C_{p}$ and $V_{p}$ are normalized poloidal circumference and valume of plasma surface.

$$
\begin{aligned}
& V_{p}=\frac{1}{2 \pi R_{0}^{3}} \int d \mathbf{r}=\int x d x d y \\
& C_{p}=\frac{1}{R_{0}} \oint d l_{p}=2 \int_{1-\varepsilon}^{1+\varepsilon}\left[1+\left(\frac{d y}{d x}\right)^{2}\right]^{1 / 2} d x
\end{aligned}
$$

The details of experimental determination of Shafranov parameter in Damavand tokamak is given is Sect. 3. By substitution of coefficients $C_{1}, C_{2}, C_{3}, C_{4}, A_{1}$ and $A_{2}$ in Eq. (12), it would be possible to determine $\beta_{p}$ and $l_{i}$ separately without using auxiliary diagnostics system such as diamagnetic flux measurements.

$$
\begin{aligned}
\beta_{p} & =\frac{2 \mu_{0}\langle P\rangle_{V}}{\left\langle B_{p}\right\rangle_{L}^{2}}=\frac{2 C_{p}^{2} A_{1}}{\left[\int\left(A_{1} x+\frac{A_{2}}{x}\right) d x d y\right]^{2}} \frac{\int \Psi(x, y) x d x d y}{V_{p}} \\
l_{i} & =\frac{\left\langle B_{p}^{2}\right\rangle}{\left\langle B_{p}\right\rangle_{L}^{2}}=\frac{(\oint d l)^{2}}{\mu_{0}^{2} I_{p}^{2}} \frac{\mu_{0}}{2 \pi} \frac{\int \psi J_{\varphi} d R d Z}{\int R d R d Z} \\
& =\frac{C_{p}^{2}}{\left[\int\left(A_{1} x+\frac{A_{2}}{x}\right) d x d y\right]^{2}} \frac{\int \psi(x, y)\left(A_{1} x+\frac{A_{2}}{x}\right) d x d y}{\int x d x d y}
\end{aligned}
$$

To find coefficients $A_{1}$ and $A_{2}$ from Eqs. (18, 19), Shafranov parameter and is obtained experimentally from well known integral model [22, 23]

$$
\Lambda \equiv \beta_{p}+\frac{l_{i}}{2}=\frac{s_{1}}{2}+s_{2}\left(1-\frac{\xi}{2}\right)
$$

where $s_{1}$ and $s_{2}$ are two experimental parameters and are represented in integral forms as

$$
\begin{aligned}
& s_{1}=\frac{1}{\mu_{0}^{2} I^{2} R_{0}} \int\left[\left(B_{\theta}^{2}-B_{r}^{2}\right) r \mathbf{n} \cdot \mathbf{e}_{\mathrm{r}}-2 B_{\theta} B_{r} r \tau \cdot \mathbf{e}_{\mathrm{r}}\right] d S_{n} \\
& s_{2}=\frac{1}{\mu_{0}^{2} I^{2}} \int\left[\left(B_{\theta}^{2}-B_{r}^{2}\right) \mathbf{n} \cdot \mathbf{e}_{R}-2 B_{\theta} B_{r} \tau \cdot \mathbf{e}_{R}\right] d S_{n}
\end{aligned}
$$

Integral Eq. (23) are taken over the measurement surface $d S_{n}=2 \pi R d l_{c}$ which is considered as an elliptical contour $l_{c}$ that is defined by parametric equations

$$
\begin{aligned}
& x \equiv \frac{R}{R_{0}}=1+\varepsilon_{c} \cos \theta \\
& y \equiv \frac{Z}{R_{0}}=\varepsilon_{c} \kappa_{c} \sin \theta
\end{aligned}
$$

where $\kappa_{c}$ and $\varepsilon_{c}=\frac{c}{R_{0}}$ are introduced as elongation factor and inverse aspect ratio parameter of the measurement surface respectively. Figure 2 shows schematic view of the measurement surface for a tokamak with elliptical cross section. Parameter $\xi$ belongs to the relative difference of the major radius $R_{0}$ which can be ignored in large aspect ratio, up-down symmetric tokamaks. To calculate $s_{1}$ and $s_{2}$, magnetic field components $B_{\theta}$ and $B_{r}$ should be measured at discrete points along the contour element $d l_{c}$ and their dependency to $\theta$ should be considered. In the case of elliptical cross section, inner products of unit vectors in integrals (23) are given as: 


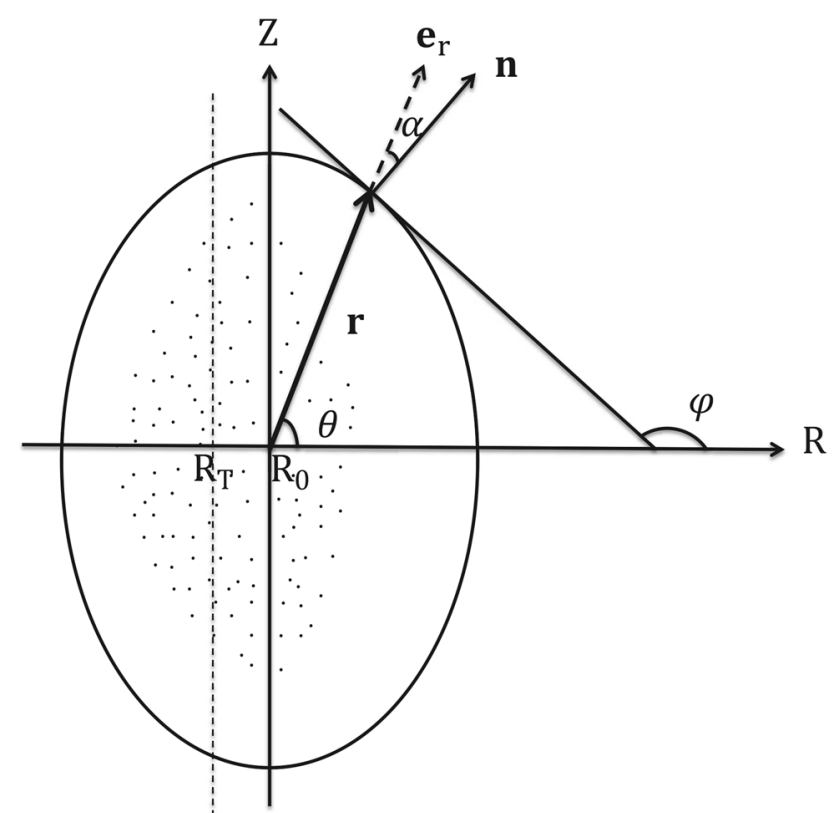

Fig. 2 Schematic of measurement surface in a tokamak with elliptical cross section

$$
\begin{aligned}
\mathbf{n} \cdot \mathbf{e}_{r} & =\cos \alpha=\frac{e^{2} \cos ^{2} \theta-1}{\sqrt{1+e^{2}\left(e^{2}-2\right) \cos ^{2} \theta}} \\
\tau \cdot \mathbf{e}_{\mathrm{r}} & =\sin \alpha=\frac{\left(\frac{e^{2}}{2}\right) \sin 2 \theta}{\sqrt{1+e^{2}\left(e^{2}-2\right) \cos ^{2} \theta}} \\
\mathbf{n} \cdot \mathbf{e}_{\mathrm{R}} & =\cos (\theta-\alpha)=\frac{\cos \theta\left(1-e^{2} \cos 2 \theta\right)}{\sqrt{1+e^{2}\left(e^{2}-2\right) \cos ^{2} \theta}} \\
\tau \cdot \mathbf{e}_{\mathrm{R}} & =-\sin (\theta-\alpha)=\frac{\sin \theta\left(2 e^{2} \cos ^{2} \theta-1\right)}{\sqrt{1+e^{2}\left(e^{2}-2\right) \cos ^{2} \theta}}
\end{aligned}
$$

where $e=\sqrt{1-\kappa_{c}^{2}} / \kappa_{c}$ is the eccentricity factor of the measurement surface and $\alpha$ denotes as normal angle which is the angle between the radius vector $\mathbf{r}$ and the normal vector n. Finding plasma current $I_{p}$ and Shafranov parameter $\Lambda$, plasma internal inductance and poloidal beta are determined separately. It is also possible to find plasma internal inductance semi-analytically using its definition (6). For this reason, the well known parabolic-like profile of the toroidal current density (appropraite for large aspect ratio, Ohmically heated tokamaks) is used as [1, 23]

$J_{\varphi}(r)=\left\{\begin{array}{l}J_{0}\left(1-\frac{r^{2}}{a^{2}}\right)^{v}, \quad r \leq a \\ 0, \quad r>a\end{array}\right.$

using the Amper's law $\frac{1}{r} \frac{d}{d r}\left(r B_{\theta}\right)=\mu_{0} J$, the poloidal magnetic field, associated to the profile of the toroidal current density, is obtained as
$B_{\theta}(r)=\left\{\begin{array}{l}B_{\theta a} \frac{a}{r}\left[1-\left(1-\frac{r^{2}}{a^{2}}\right)^{v+1}\right], \quad r \leq a \\ B_{\theta a} \frac{a}{r}, \quad r>a\end{array}\right.$

where $B_{\theta a}=\frac{\mu_{0} J_{0}}{2(v+1)}=\frac{\mu_{0} I_{p}}{\oint d l_{p}}$ and $a$ are the poloidal magnetic field and minor radius on the plasma surface. Using Eq. (6) and (27), plasma inductance is found as

$l_{i}=\frac{4 \kappa}{1+\kappa^{2}}\left[\alpha+\ln \left(\frac{c}{a}\right)\right]$

where $\kappa$ is the elongation factor of the plasma column, $c$ defines the minor radius of the measurement surface and $\alpha$ is a constant which is determined by parameter $v$. Finding evolution of $\Lambda$ and $l_{i}$ form Eqs. (22) and (28), qualitative evolution of the plasma energy confinement time can be found in term of $\beta_{p}$ using energy balance Eq. (1).

\section{Analysis of experimental measurements}

Magnetic fields measurements are required to find the evolution of Shafranov parameter, plasma internal inductance and the energy confinement time in Damavand tokamak which is a small size, large aspect ratio $\left(\varepsilon=\frac{a}{R_{0}} \approx 0.2\right.$ ), non-circular tokamak. The maximum elongation factor and minor radius (limiter radius) of the tokamak are $\kappa \approx 1.41$ and $a_{0}=7 \mathrm{~cm}$, respectively. The maximum current and discharge duration of the plasma are $35 \mathrm{kA}$ and $25 \mathrm{~ms}$, respectively. A set of radial (normal) and poloidal (tangential) magnetic probes are distributed along the measurement surface are shown in Fig. 3. In Fig. 4 temporal variation of the plasma current, toroidal magnetic field and loop voltage are shown during a typical shot of Damavand tokamak. In large aspect ratio tokamaks, magnetic fields $B_{\theta}$ and $B_{r}$ can be analyzed using Fourier expansion $[8,9]$

$$
\begin{aligned}
& B_{\theta}(r, \theta)=B_{c}\left[1+\sum_{k=1} \lambda_{k} \cos k \theta\right] \\
& B_{r}(r, \theta)=B_{c} \sum_{k=1} \mu_{k} \sin k \theta
\end{aligned}
$$

where $B_{c}$ is the poloidal magnetic field on the measurement surface. Fourier coefficients $\lambda_{k}$ and $\mu_{k}$ are obtained from experimental data acquired from poloidal and radial magnetic probes which are mounted around the plasma. 


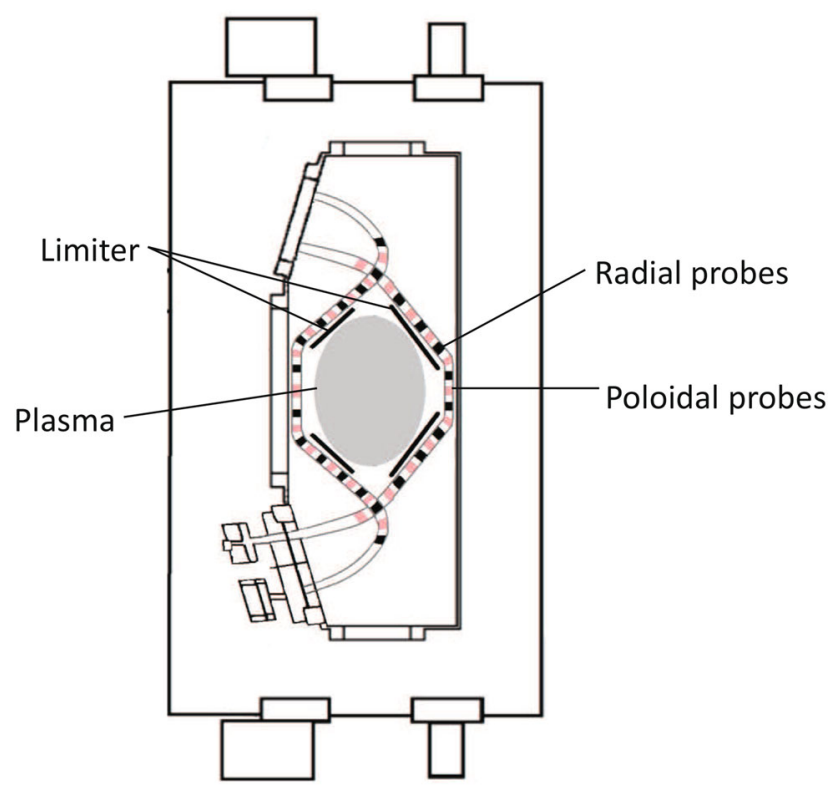

Fig. 3 Schematic cross sectional view of magnetic probes location in Damavand tokamak
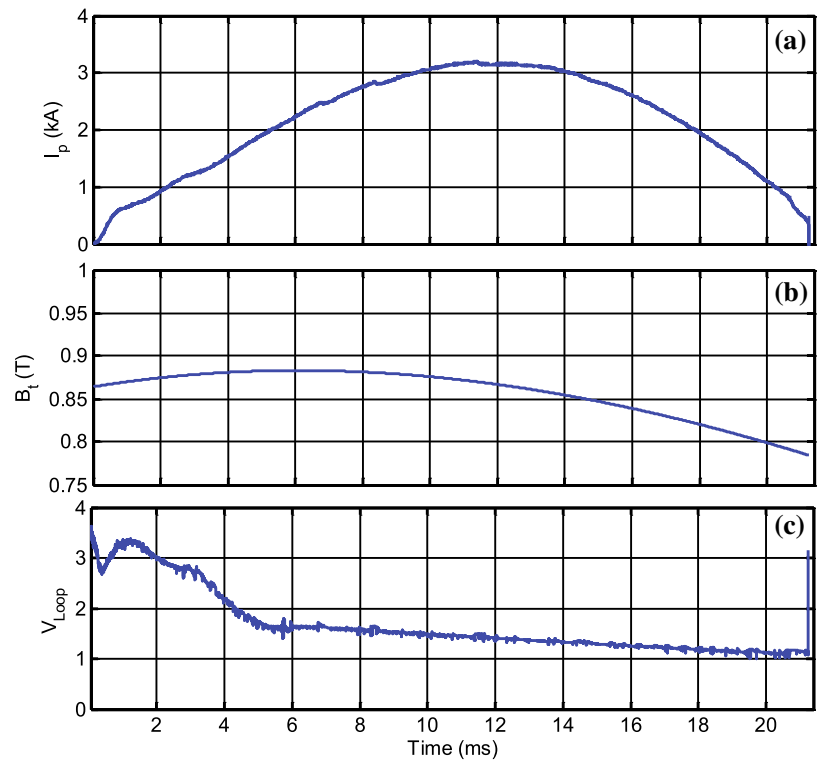

Fig. 4 Plasma current (a), toroidal magnetic field (b) and loop voltage (c) in a typical shot of Damavand tokamak

$$
\begin{aligned}
\lambda_{k} & \equiv \frac{4}{N B_{c}} \sum_{j=1}^{N} B_{\theta}\left(r, \theta_{j}\right) \cos k \theta_{j} \\
\mu_{k} & \equiv \frac{4}{N B_{c}} \sum_{j=1}^{N} B_{r}\left(r, \theta_{j}\right) \sin k \theta_{j}
\end{aligned}
$$

For elliptical plasma surface with enough symmetry, it is reasonable to retain first and second harmonics of the Fourier expansion. The induced voltage in a probe of turns $n$ per unit length and area $A$, from the variable magnetic field produced from current $I$ can be written as

$$
V_{i n}=-n A \frac{d B(t)}{d t}
$$

Therefore, the magnetic field induced in a probe can be expressed in term of output voltage

$$
B(t)=-n A \int V_{\text {in }}(t) d t=k V_{\text {out }}
$$

where $k$ is a constant parameter that depends on cables resistance, integrator time scale and geometrical parameters of the magnetic probes. Using empirical definition of the plasma internal inductance (6), parameter $v$ can be determined analytically, and therefore, the zeroth order of the current density and poloidal magnetic field are found in term of v. Fig. 5. shows radial variation of the toroidal current density and poloidal magnetic field at different times of the discharge. Evolution of Shafranov parameter and semi-analytical expression of the plasma internal inductance (Eq. 28) are shown in Fig. 6. It is found that the Shafranov parameter has almost uniform profile during the plasma discharge. Fig. 7 shows results of parametrization method (analytical solution of the GSE) in determination of the poloidal beta and plasma internal inductance. It is found from Fig. 7b that the GSE approach has good agreement with the semi-analytical expression of the plasma internal inductance (Eq. 28) in flat-top, steady state regime of the plasma current. Fig. 8 represents evolution of the Ohmic input power, total thermal energy and energy confinement of Damavand tokamak. The maximum energy confinement time which corresponds to the minimum microinstabilities, collisions and transport of the plasma is about $2.5 \mathrm{~ms}$ and takes places at $12.3 \mathrm{~ms}$ time of the discharge where the maximum input power is $53 \mathrm{~kW}$.

\section{Conclusion}

Output signals of magnetic probes, measuring normal and tangential components of poloidal magnetic field, were used to determine poloidal beta and plasma internal inductance in a regular discharge of Damavand tokamak. Linear combination of plasma internal inductance with poloidal beta $\left(\beta_{p}+\frac{l_{i}}{2}\right)$ was determined from integral representation of poloidal magnetic field components, evolution of the poloidal beta and plasma internal inductance was found using analytical solution of Grad-Shafranov equation and compared with semi-analytic expression of the plasma internal inductance which is based on definition of parabolic-like profile of the toroidal current density. Finding poloidal beta, input power time (energy 

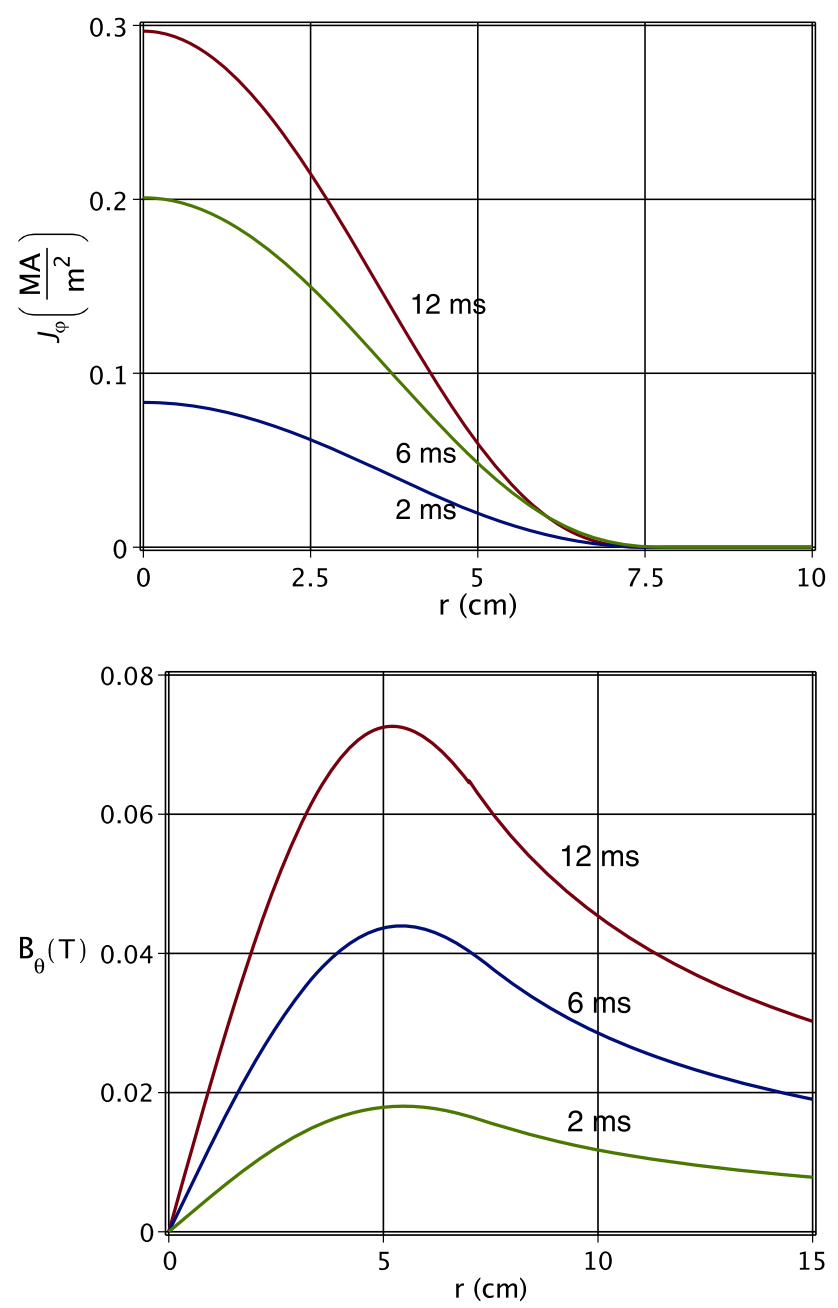

Fig. 5 Radial variation of toroidal current density and poloidal magnetic field at different times of the Damavand tokamak discharge
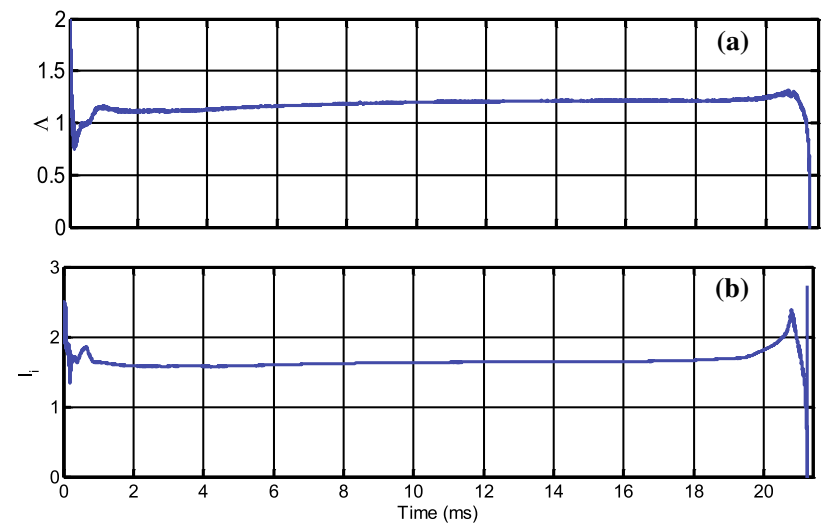

Fig. 6 Temporal variation of Shafranov parameter (a) and semianalytical expression of plasma internal inductance (Eq. 28) (b)

confinement time) of Damavand tokamak was determined qualitatively in terms of poloidal beta using power balance equation. (a) 0.8
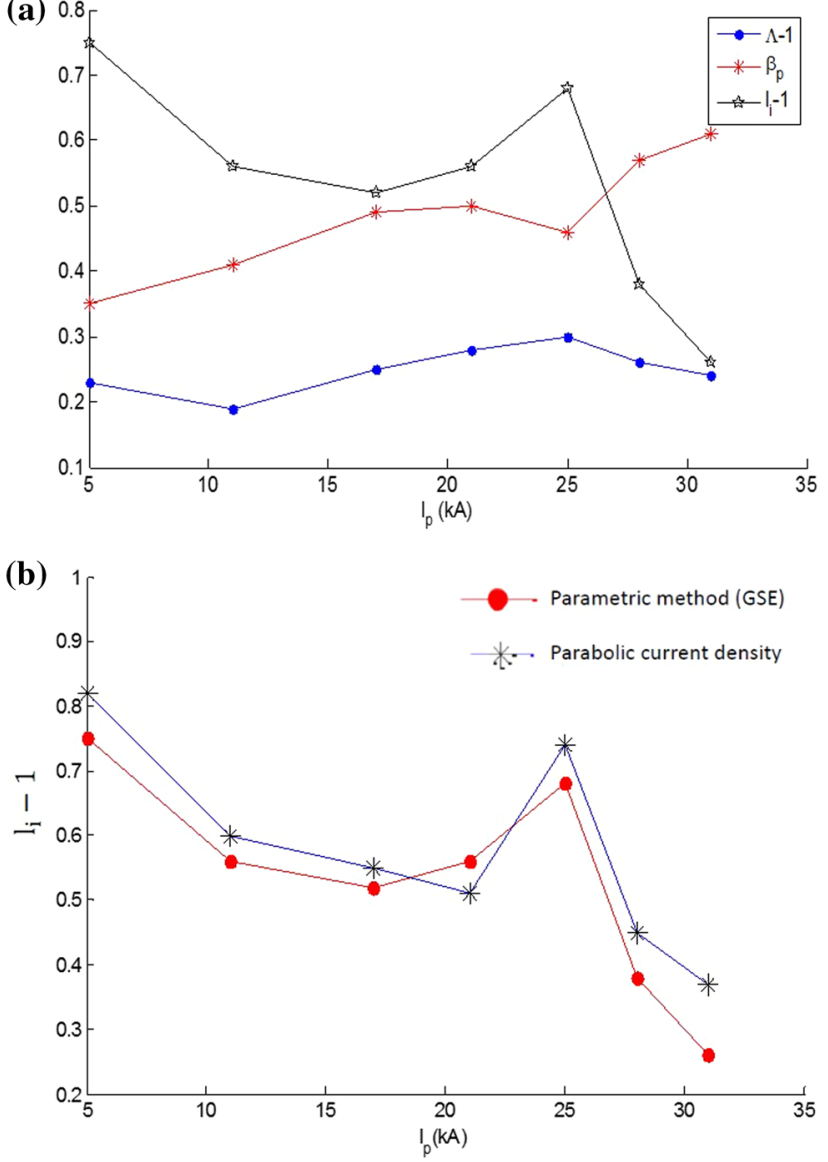

Fig. 7 Determination of poloidal beta and internal inductance using parametrization method (analytical solution of GSE) (a) and comparison of the plasma internal inductance with the semi-analytic expression (Eq. 28) (b)

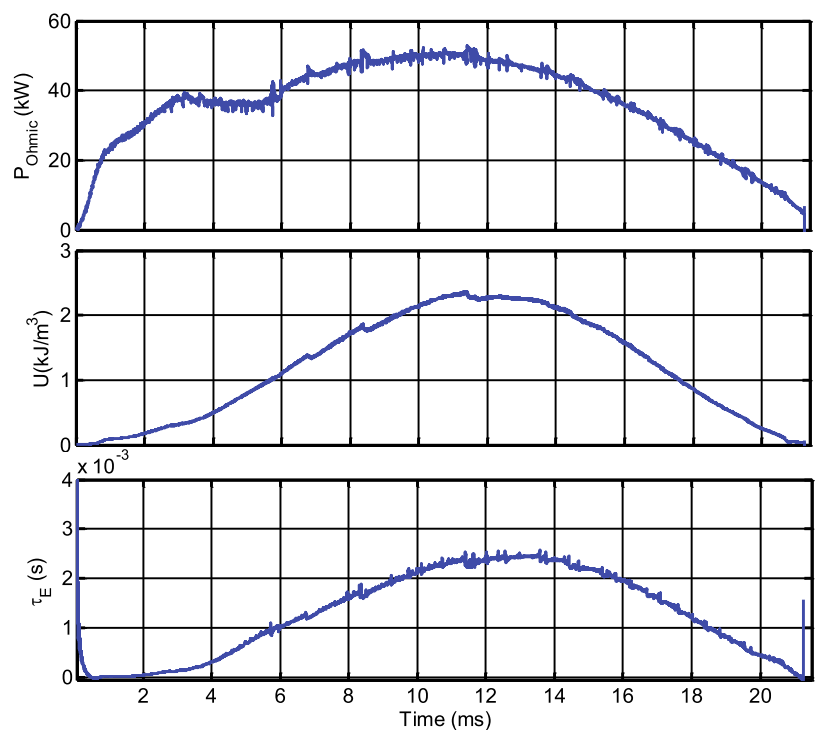

Fig. 8 Evolution of input Ohmic power (c), total thermal energy (b) and energy confinement time (c) 
Acknowledgments The authors would like to thank Damavand tokamak scientific group and Prof. M. Ghoranneviss, the head of the Plasma Physics Research Center (PPRC), for their experimental and theoretical support of the research.

Open Access This article is distributed under the terms of the Creative Commons Attribution 4.0 International License (http://crea tivecommons.org/licenses/by/4.0/), which permits unrestricted use, distribution, and reproduction in any medium, provided you give appropriate credit to the original author(s) and the source, provide a link to the Creative Commons license, and indicate if changes were made.

\section{References}

1. W. M. Stacey, Fusion Plasma Physics (Wiley-VCH., 2005)

2. K. Miyamoto, Plasma physics and controlled nuclear fusion (Springer, 2006)

3. Yong, S., et al.: Plasma Sci. Technol. 11, 131 (2009)

4. Elahi, A.S., Ghoranneviss, M.: Phys. Scr. 81, 055501 (2010)

5. Elahi, A.S., et al.: J. Fusion Energ. 28, 346 (2009)

6. M. Ariola, and A. Pironti, Magnetic Control of Tokamak Plasmas (Springer, 2008)

7. Elahi, A.S., et al.: J. Fusion Energ. 28, 385 (2009)
8. Ninomiya, H., Suzuki, N.: Jpn. J. Appl. Phys 21(9R), 1323 (1982)

9. Shen, B., et al.: Rev. Sci. Instrum. 78, 093501 (2007)

10. Noori, E., et al.: Phys. Scr. 90, 105604 (2015)

11. A. Rahimi-Rad, et al., Phys. Scr. 86, 01550186 (2012)

12. Alladio, F., Crisanti, F.: Nucl. Fusion 26, 9 (1986)

13. Elahi, A.S., Ghoranneviss, M., Trans, I.E.E.E.: Plasma Sci. 38 , $181(2010)$

14. Elahi, A.S., Ghoranneviss, M., Trans, I.E.E.E.: Plasma Sci. 38, 3163 (2010)

15. Lao, L., et al.: Nucl. Fusion 25, 11 (1985)

16. Elahi, A.S., Ghoranneviss, M.: Phys. Scr. 80, 055502 (2009)

17. Elahi, A.S., Ghoranneviss, M., Trans, I.E.E.E.: Plasma Sci. 41, 334 (2013)

18. J. P. Freidberg, Ideal magnetohydrodynamics (Plenum Publishing Company , 1987)

19. I. H. Hutchinson, Principles of Plasma Diagnostics (Cambridge University Press, 2005), pp. 10-33

20. S.B. Zheng, A.J. Wootton and E.R. Solano, Phys. Plasmas. 3, 1176 (1196)

21. S. Abdullaev, Magnetic stochasticity in magnetically confined fusion plasmas (Springer, 2014), pp. 20-27

22. Shafranov, V.: Plasma Physics 13, 9 (1971)

23. J. Wesson, Tokamaks (Oxford University Press, 2011), pp. 502505 